\title{
UK neurology response to the COVID-19 crisis
}

\author{
Authors: Catherine J Mummery ${ }^{A}$ and Christopher M Kipps ${ }^{B}$ on behalf of the Association of British Neurologists
}

COVID-19 has led to seismic changes in neurological practice in a matter of weeks. The Association of British Neurologists has supported neurology specialists and patients during this rapid reorganisation and its attendant challenges. We have written guidance on structured service transformation, considering the need to sustain long term care while responding to acute developments; we have recognised that staff experience differs and that this, as well as individual risk factors should be considered when redeployment occurs. Appreciating that there may be understandable anxiety when facing a working routine outside normal practice, we have signposted ethical and psychological support for individuals. We have also focused on our patients: we have facilitated a national alert system to register all neurological COVID cases, coordinating research efforts on this new disease; finally we have defined how to identify the most vulnerable patients under our care. When this initial wave of the pandemic subsides, we will have planned for return to the new 'norm', ready to embrace innovation where appropriate, aiming to minimise fall-out in our chronic disease population, and potentially having enhanced and modernised our services.

KEYWORDS: COVID-19, neurology, service reconfiguration, service transformation, workforce deployment

DOI: $10.7861 /$ clinmed.2020-0159

\section{Introduction}

The COVID-19 pandemic has led to wholesale changes in clinical pathways and means of assessment, implemented within the space of a few weeks. Neurologists have had to rapidly adapt to new ways of working. Outpatient clinics are now almost entirely conducted by phone or video link, and direct physical examination is minimised unless essential for patient care decisions. Direct neurological support to the emergency department is the default position.

Authors: ${ }^{A}$ consultant neurologist and honorary associate professor, National Hospital for Neurology and Neurosurgery, University College London Hospital NHS Foundation Trust and University College London, London, UK; ${ }^{\mathrm{B}}$ Consultant neurologist and honorary senior clinical lecturer, University Hospital Southampton NHS Foundation Trust and University of Southampton, Southampton, UK
In response to the developing crisis, the Association of British Neurologists (ABN) has produced COVID-19-related guidance internally, and with partners such as the Royal College of Physicians (RCP), to provide support to our members and our patients. The ABN's structure has proved nimble, with input from our disease-specific expert advisory groups and highly engaged members enabling rapid collection, processing and dissemination of information. This article sets out the work done so far, recognising that as the situation continues to evolve and accelerate, we must remain in response mode, adjusting advice when necessary. At the same time, an eye to the future is needed to maintain critical non-COVID services and build on the accelerated innovation seen in recent weeks.

\section{Key points}

It is essential to provide an agile and high-quality response to those acutely affected.

Exacerbations of long-term conditions and the effects of therapy continue to occur and must be addressed.

Effective outcomes are needed in the midst of rapid decision-making.

A measured, graded escalation is key, rather than an all-in-one 'knee-jerk' response, while flexibility is required due to the unstable and changing nature of the crisis.

Multiple levels of organisation need to be considered.

Local variation is expected and appropriate.

Clarity is needed on standards, governance and quality of decision-making.

\section{Rapid reorganisation of neurology services}

The COVID-19 pandemic requires rapid reorganisation of services at multiple levels. It is essential to treat people who are acutely affected promptly, yet at the same time to maintain the best possible quality of service provision for non-COVID-19 patients presenting during this crisis. Urgent neurology cases will still occur during this period; effective management of this group of patients helps reduce capacity pressures on the NHS, prevents unnecessary morbidity and mortality, and reduces the likelihood of excess case load or increase in morbidity when services start returning to normal.

Reorganisation must be rapid, but measured in response, tailored and responsive to the level of need at each stage. For example, 
halting all elective outpatient work to deploy all consultants to acute rotas from the outset fails to take into account the time lag during which numbers of affected people increase slowly, or the ongoing needs of the existing neurological population.

National guidelines provide a framework for action, but at a local level escalation of response will vary depending on the population. Certain areas of the country with high population densities, such as London and Birmingham, have been affected earlier and more severely. Previously collected data show that the organisation of acute, inpatient and outpatient neurological services varies widely across the UK. The variability in service organisation and level of impact will affect local planning through the different stages of the pandemic. Therefore, regional variations in approach, timing and resources are expected and appropriate.

\section{Structured escalation of response}

During the preparation phase, it has been helpful to identify different escalation levels that help define specific actions, as well as minimum service levels based on availability of staff and capacity of the hospital. Importantly, these tools can be used in both escalation and de-escalation phases and can be utilised within a service (or even subspecialty) in response to service pressures that may not be experienced uniformly across a trust, a region or the NHS as a whole. It allows a degree of flexibility which might otherwise be lost (Fig 1). For example, using a red-ambergreen (RAG) rating scheme:

$>$ Blue-normal clinics

$>$ Green - prepare - triage clinics

$>$ Amber - no face-to-face clinics unless urgent

$>$ Red-no clinics - urgent only

> Black - treatments for life-threatening conditions only

\section{Minimum levels of service need definition}

During this period, the ongoing need for non-COVID-19 neurological services will be pressured by the redeployment of staff and the limited ability to perform diagnostics. There is a recognition that while the aim is to maintain a service, the risk threshold in diagnostic decision making has altered and the standard levels of monitoring may be difficult to provide. Neurology practice and recommendations must be sensible and practical, tailored to circumstances, avoiding use of highrisk medications if they can be put off until de-escalation, and providing clear recommendations to patients on when they need to seek help. In some situations it may be reasonable, and in fact desirable, to suspend routine follow-up, but to protect rapidresponse 'SOS' clinics via phone to ensure patient safety. In acute diagnostic pathways, the early involvement of a consultant neurologist will reduce admission numbers, investigations requested and length of stay. Expert input at the point of entry to the hospital system provides confidence for early discharge in common neurological conditions such as headache, migraine, sensory disturbance, black-outs, single seizures and stroke mimics. Accurate early diagnosis is facilitated, which may accelerate appropriate treatment and avoid high dependency and ITU admissions in disorders such as Guillain Barré syndrome, myasthenia gravis, encephalitis, or unstable epilepsy. Importantly, there is growing evidence of neurological presentation in a proportion of patients with COVID-19 infection ${ }^{1}$ and the capacity to identify and support such patients is vital.

In addition to those acutely affected, it is vital that management of long-term neurological conditions is sustained. Involvement of neurology specialists, including neurologists, specialist nurses, neuropharmacists and specialist allied healthcare professionals remains crucial in continuing support for patients and their

\section{Outpatient activity normal \\ $>$ Advice and guidance offered where appropriate \\ Blue $>2 \mathrm{WW}$, urgent and routine clinics run as normal \\ > MDT for complex patients as standard (virtual or in person) \\ Full range of diagnostic and therapeutic options available}

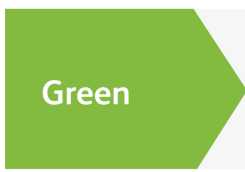

Prospective triage of patients (excluding $2 \mathrm{WW}$ and known high risk urgent groups)

$>$ Clinics run normally. Higher level of 'did not attend' anticipated. Risk-based review of therapies

$>$ Consultants begin triage of all routine booked appointments 2 weeks ahead. Organised via clinical leads with approach agreed for each subspecialty

> Move to non-face-to-face where possible, including MDT

$\begin{array}{ll}\begin{array}{l}\text { Non-face-to-face by default } \\ >\end{array} & \text { All patients converted to advice and guidance service or telephone appointment (or video where already available), with the } \\ & \text { exception of already-known high risk groups. Virtual MDT only }\end{array}$

Urgent outpatients only
$>$ Definition agreed by clinical leads. All others deferred. Medical staff re-prioritised to cover depleted workforce. Patients moved into
$\quad$ daily urgent clinics
$>$ All patients in routine slots postponed (accepting risk in incorrect coding)
$>$ Urgent patients triaged if necessary

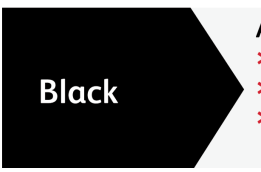

Admission avoidance only

$>$ Inadequate clinical workforce to maintain service OR staff re-prioritised to cover depleted workforce

All neurology outpatients deferred

Outpatient estate used for admission avoidance/assessment areas

Fig 1. RAG rating framework for service provision. $2 \mathrm{WW}=$ two week wait pathway; MDT = multidisciplinary team meeting. 
families/carers in the community to avoid deterioration and admission with common problems such as falls, urinary tract infections and respiratory infections. A significant proportion of patients are on immunosuppressive therapy; as a specialty we must identify vulnerable individuals and ensure they have the appropriate advice and support. The effects of social distancing and self-isolation in vulnerable patients with long-term conditions may be marked, with particular burden placed on caregivers. With disruption in the normal ways of delivering care, a lack of guidance on minimum standards and governance expectations risks inadequate care provision for the most vulnerable.

$A B N$ guidance for those with neurological conditions is accessible here at www.theabn.org/page/covid19_response.

\section{Workforce deployment}

Redeployment of clinicians is expected, but should take into account factors such as individual work experience and health risks. A degree of flexibility is necessary due to the uncertainties inherent in an evolving crisis situation.

The RCP has issued guidance that 'no one should work outside their competence, though many will be working outside their comfort zone'. This impacts on trainees but also on clinicians who may not have done acute medicine for some time. All neurologists have completed general medical training to MRCP level, but they do not as a rule continue in general medicine after joining specialist training. Some, but not all, will have ongoing or past stroke experience.

In addition, risk factors such as age and presence of health issues need to be taken into account. When planning, sensitivity is required to the possibility of inadvertently unmasking a colleague's health status, and recognising that there are multiple ways of supporting the COVID-19 response, many of which do not involve frontline tasks.

We have recommended a tiering system for neurologists in which:

> neurologists with recent stroke experience focus efforts on TIA clinics and stroke management

> neurologists with less stroke experience staff phone clinics, emergency review, ward attendee system

> neurologists with risk-associated health conditions, receiving immunosuppressive therapies or aged over 55-60 offer support via remote working on triage or telemedicine clinics

> registrars move to acute neurological rota, stroke service and later general acute medicine

$>$ those with specific skills should be utilised for high-demand areas such as the intensive care unit (ITU).

Specialist nurses are vital for the ongoing support of patients with long term conditions such as multiple sclerosis, epilepsy, Parkinson's disease and neuromuscular conditions. It is inevitable that many will be redeployed to clinical acute care, but a minimum level of support must be specified for each service to ensure patients who are already feeling isolated and vulnerable have continuity in their support.

We have recommended specialist nurse requirements are tiered in a similar way to neurologists, so that:

> nurses with risk-associated health conditions, receiving immunosuppressive therapies or aged over 55-60 refocus on remote working, telemedicine clinics and liaison with primary care to maintaining support for patients with long term conditions

\section{Box 1. Staffing change examples}

> All consultants with predominantly DGH sessions released from roles in neurosciences centres

> Accordingly, stroke and neurology on-call rota revised - stroke and daytime neurology services staffed by neuroscience centre internally

> Individual sub-specialties provide core and urgent services with, for example, daily multiple sclerosis consultant service to deal with any issues as they arise. Home delivery for immunotherapies where possible

> Combined stroke and neurology wards, run by neurology to release stroke team; beds used flexibly

$>$ Increased community support to speed up discharge in stroke and rehabilitation

$>$ Roving physician colleagues to provide help and support to neurologists in general medicine roles

> Daily consultant-led clinic focusing jointly on transient ischemic attack and hot neurology, including emergency department in-reach, inpatient referrals and urgent GP calls

$>$ nurses with recent acute experience redeploy to the acute ward or ITU.

Some nurses may have been part re-deployed, which partly eases the pressure. Where possible, some specialist nurse activity has been protected despite part-time re-deployment to other areas.

A support system for those being redeployed to a new area is vital with retraining, and expert advisors will be needed on hand 24/7.

When considering deployment, size and complexity of the neurological team is also a factor: the larger staffing complement in neurosciences centres helps to maintain specialist care, while in DGHs, neurologists have been deployed earlier to general medicine rotas. With appropriate negotiation, this has ensured neurosciences centres take on responsibility for ongoing regional care, despite local level suspension of services (Box 1).

\section{Enhanced and accelerated communication}

Effective channels of communication have been vital in keeping abreast of a rapidly changing crisis and ensuring advice remains current.

> Inter-organisational - chains of communication have been enhanced between external organisations such as the RCP, $\mathrm{NHS}$ England and the $\mathrm{ABN}$, facilitating quick turnaround of continuously evolving guidelines.

Reduction of service provision, and return of care to the community requires clear, accurate and accessible information at a time of competing demands and rapid change.

> Primary care - responsive pragmatic discussions are necessary to prevent admission and facilitate discharge; there should be clear communication of what is and what is not possible.

$>$ Inter-regional - there should be flexible decision-making on prioritisation of direct clinical care between DGHs and neuroscience/neurology centres, relating to the distribution 
both of patients (eg one London trust taking on pan-London neurooncology to free up space in other sites with higher COVID demand) and of staff (eg releasing linked hospital staff to the site requiring most clinical support).

> Interdisciplinary - stroke medicine, general medicine and neurology need rapid concise discussion on deployment to point of need.

It is vital at such a time of uncertainty that we maintain close contact with our patient population.

> Patient cohort identification: a national surveillance study, 'CoroNerve', has been facilitated via the ABN RADAR platform to collect information on neurological manifestations of COVID-19.

> Vulnerable patient protection: we have contacted vulnerable patients, and have encouraged self-registration and shielding.

> Charitable organisations: we have advised groups such as the Neurological Alliance and work together with them on documentation for patients.

\section{With new ways of working, support for practice is needed}

Enhanced communication with primary and secondary care is fundamental, with dedicated consultant time offered for GP liaison to prevent emergency admission. 7-day working is the norm. There has been a major shift in work patterns, with some freed up from outpatient time to plan, and others, including those that can only work remotely, backfilling roles that can now be performed off-site. This has helped increase acute responsiveness in a limited workforce.

Risk management is different to what is seen in normal times, yet accountability remains. A range of supporting tools have been developed to facilitate the change in practice and made available on the ABN website (Box 2). Priority was given to developing resources that are directly useful for clinical decision making (such

Box 2. Online resources from the Association of British Neurologists

Resources supporting practice change and risk profiling

$>$ Emergency triage tool

> Advice on conducting phone and video consultations and digital care provision

$>$ Definition of high-risk groups in neurological conditions

$>$ Multiple sclerosis: DMT drug guidance

$>$ Advice and guidance information sheets

$>$ Reporting tool for suspected neurological complications of COVID (RADAR)

Resources to support ethical decision-making and staff wellbeing

$>$ Links to ethical guidance

> Links to personal protection (national, appreciating local variation)

$>$ Wellbeing support links

$>$ Advice forum (blog)

These resources are available on the $\mathrm{ABN}$ website (www.theabn.org). as risk profiling) and changed practice (such as triage or advice and guidance)

\section{New ways of working - support for wellbeing}

Rapid change and significant uncertainty is stressful, with individuals asked to work in areas they are unfamiliar with, in a situation that increases professional and personal risk. Dealing with anxious patients both with and without COVID infection exacerbates these stresses as services come under pressure and those that rely on community support are increasingly isolated. As a result, support for clinicians has been a strong focus (Box 2).

\section{The end of the crisis - returning and reflecting}

The COVID pandemic will end, but how and when remains unclear. There may well be a graded re-opening of services as capacity pressures ease and the need to re-focus on non-COVID neurological patients becomes more pressing. During this process, local fluctuations in service pressure may well occur, and ongoing flexibility in terms of local practice is likely to be needed for some time. As with the gradual increase in social freedoms, we will need to plan our de-escalation. Services will transition back from skeleton to fully staffed and we will need to re-establish elective assessment, investigation and therapeutics, dealing with any accumulated backlog.

When this occurs we must take what we have learned from this process and integrate it with the best from our previous systems. Things will have changed in ways barely imaginable a matter of only weeks or months previously. Some of this will be for the better, and we may wish to maintain some changes, having seen what is possible. We may also notice better responsiveness and greater integration with our acute medical colleagues.

The 'new norm' is likely to include more telemedicine, greater reliance on digital tools, and more flexibility about how we conduct clinics, which patients we see and who we should follow up over time. We will have gained skills in providing advice and guidance, and become very focused on the essential. The wholesale changes in our methodology over such a short period of time will have had unintended consequences, with opportunities as well as risks. We have discussed for decades some of the changes that have been wrought in a matter of days.

\section{Acknowledgements}

We would like to acknowledge the enormous amount of work done by Jon Sussmann, honorary secretary to the ABN, much of which is referenced in this paper. In addition, we acknowledge the University Hospital Southampton COVID Outpatients project team for work on the RAG rating triage tool, which we have adapted.

\section{Reference}

1 Mao L, Jin $\mathrm{H}$, Wang M et al. Neurologic manifestations of hospitalized patients with coronavirus disease 2019 in Wuhan, China. JAMA Neurol 2020, in press (doi: 10.1001/jamaneurol.2020.1127).

Address for correspondence: Dr Catherine Mummery, Dementia Research Centre, National Hospital for Neurology and Neurosurgery, Box 16 Queen Square, London WC1N 3BG, UK.

Email: cath.mummery@nhs.net 